\title{
Dissociation of $\mathrm{O}_{2}$ at $\mathrm{Al}(111)$ : The Role of Spin Selection Rules
}

\author{
Jörg Behler, ${ }^{1}$ Bernard Delley, ${ }^{2}$ Sönke Lorenz, ${ }^{1}$ Karsten Reuter, ${ }^{1}$ and Matthias Scheffler ${ }^{1}$ \\ ${ }^{1}$ Fritz-Haber-Institut der Max-Planck-Gesellschaft, Faradayweg 4-6, D-14195 Berlin, Germany \\ ${ }^{2}$ Paul-Scherrer-Institut, HGA/123, CH-5232 Villigen PSI, Switzerland
}

(Received 28 October 2004; published 25 January 2005)

\begin{abstract}
A most basic and puzzling enigma in surface science is the description of the dissociative adsorption of $\mathrm{O}_{2}$ at the (111) surface of Al. Already for the sticking curve alone, the disagreement between experiment and results of state-of-the-art first-principles calculations can hardly be more dramatic. In this Letter we show that this is caused by hitherto unaccounted spin selection rules, which give rise to a highly nonadiabatic behavior in the $\mathrm{O}_{2} / \mathrm{Al}(111)$ interaction. We also discuss problems caused by the insufficient accuracy of present-day exchange-correlation functionals.
\end{abstract}

DOI: 10.1103/PhysRevLett.94.036104

Oxygen-metal interactions are responsible for everyday phenomena such as corrosion and form the atomic-scale basis behind numerous technological applications such as oxidation catalysis. It is therefore most discomforting that, despite several decades of research in surface science, the initial step in the oxygen-metal interaction, namely, the dissociation process of $\mathrm{O}_{2}$ molecules over metal surfaces, is not yet understood. This is, in particular, so for what is often called the most simple metal surface, namely, $\mathrm{Al}(111)$ : a close-packed surface of a nearly free electron metal. For the initial interaction of $\mathrm{O}_{2}$ with $\mathrm{Al}(111)$, experiments have consistently shown [1,2] that the initial dissociative sticking probability for thermal $\mathrm{O}_{2}$ is very low (about 2\%). Density-functional theory (DFT) calculations, on the other hand, found that dissociation is not hindered by energy barriers [3], which implies that the initial sticking coefficient should be very high (about $100 \%$ ). Another intriguing aspect of the $\mathrm{O}_{2} / \mathrm{Al}(111)$ system is that at very low coverages the distribution of adsorbed oxygen atoms is random, even when adsorption is performed at temperatures at which thermal diffusion cannot play a significant role [2]. Thus, it is impossible to trace back which two adatoms stem from the same molecule. Initially, this led to the suggestion that the adsorption energy is used to trigger the diffusion of "hot adatoms" [2]. More recently, a different explanation has been suggested ("abstraction"), where only one $\mathrm{O}$ atom is adsorbed and the other one is repelled back into the vacuum [4]. Again, theoretical work, so far, does not give a clue why this may be so. Thus, one may ask, what we can trust in surface science when the understanding of a most basic and simple system for molecule-surface interactions is so clearly lacking.

Figure 1 summarizes the experimental data for the initial sticking coefficient as a function of the kinetic energy of incoming $\mathrm{O}_{2}$ molecules for a molecular beam at normal incidence (solid diamonds) [1], as well as the result of what has hitherto been the standard theoretical treatment (labeled as "theory adiabatic"). Also shown is the result of the approach taken in the present Letter (labeled as "theory triplet"), which will be detailed below. Obvi-
PACS numbers: 82.20.Kh, 68.35.Ja, 82.20.Gk

ously, there is hardly any similarity between the theoryadiabatic curve and the experimental result. Though we called this the "standard theoretical treatment," we note that already the calculations behind the theory-adiabatic curve (and also behind the theory-triplet curve) are much more elaborate and advanced than typical approaches to obtain the initial sticking coefficient: All theoretical results presented in this Letter were obtained from extensive allelectron DFT calculations using the $\mathrm{DMol}^{3}$ code [5]. This provided the six-dimensional potential-energy surface (PES) for the $\mathrm{O}_{2} / \mathrm{Al}(111)$ system at more than 1500 geometries of the two oxygen atoms, keeping the substrate frozen. These PES data points were then interpolated by a neural network [6,7], enabling us to perform molecular dynamics (MD) calculations for about 100000 trajectories, including all possible initial molecular orientations. Thus, this approach [8] grants controlled and good statistics, in contrast to "on-the-fly $a b$ initio MD," which gives (for a frozen substrate) the same trajectories, but for which due to the high CPU cost at best only $\sim 50$ trajectories could be performed even on today's biggest computers.

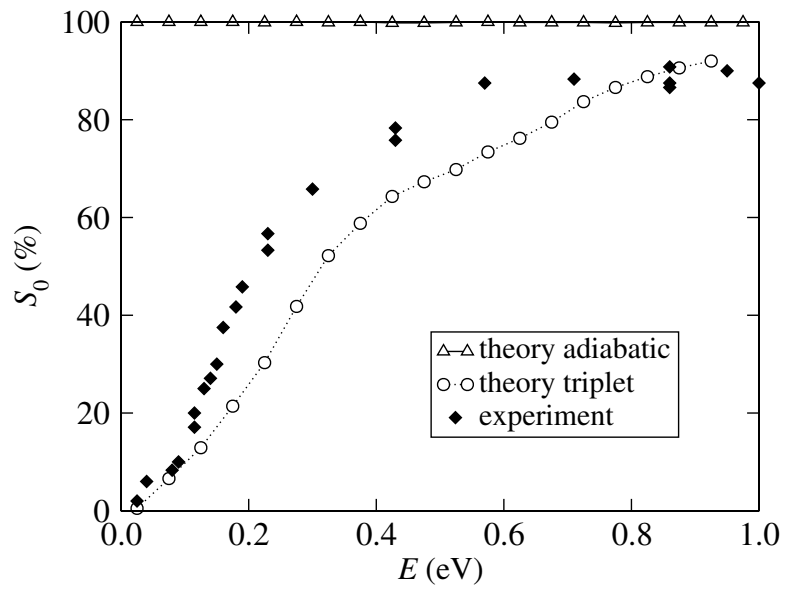

FIG. 1. Initial sticking curve of $\mathrm{O}_{2}$ at $\mathrm{Al}(111)$, based on the adiabatic (open triangles) and the spin-triplet (open circles) potential-energy surfaces using the RPBE functional. The experimental data (solid diamonds) are from Ref. [1]. 
Still, on-the-fly $a b$ initio MD has the advantage that it can also be used beyond the frozen substrate approximation. To check the validity of our treatment, we therefore performed $24 a b$ initio MD runs, where the full dynamics of the Al surface atoms was taken into account. These studies show that the adsorption energy is efficiently transferred to strong surface vibrations, and that the oxygen adatoms do not move far. Thus, the hot adatom concept is not supported. In all studied trajectories the $\mathrm{Al}(111)$ surface was affected only when the $\mathrm{O}_{2}$ was quite close to the surface, i.e., when O-Al bonds were being formed and the $\mathrm{O}-\mathrm{O}$ bond notably weakened (at molecule-surface distances below $\approx 2.5 \AA$ ). Before this point, the $\mathrm{O}_{2}$ trajectories were not changed by the substrate vibrations, and, in particular, all incoming $\mathrm{O}_{2}$ molecules were found to dissociate, fully confirming the adiabatic result shown in Fig. 1. We also performed a systematic comparison using different exchange-correlation (XC) functionals, including the Perdew-Burke-Ernzerhof (PBE) [9] and revised PBE (RPBE) [10]. The resulting PESs look different in some details; however, the resulting sticking curve is always essentially the same as the theory-adiabatic curve in Fig. 1. Hence, neither the approximate XC treatment nor the frozen substrate approximation can account for the dramatic disagreement between the theoretical and experimental results. We therefore conclude that the origin must be more fundamental, namely, in the assumed adiabatic description, restricting the impinging molecule to the electronic ground state of the combined $\mathrm{O}_{2} / \mathrm{Al}$ system at each point of the $\mathrm{O}_{2}$ trajectory. Based on less rigorous studies, this had been suggested previously $[11,12]$.

Inspecting the six-dimensional adiabatic PES reveals immediately an obvious flaw of the adiabatic description, independent of the employed XC functional: Even at the largest distances the electron chemical potentials of the $\mathrm{O}_{2}$ molecule and the $\mathrm{Al}(111)$ surface align, which is achieved by some electron transfer towards the $\mathrm{O}_{2}$ molecule. Obviously, in reality charge transfer will occur only when the two systems are getting close for a sufficiently long period of time. Recently, Hellman et al. [11] considered the influence of charge transfer by employing an approach where they replaced the $\mathrm{Al}(111)$ surface by jellium and treated the kinetic-energy operator in the Thomas-Fermi-Weizsäcker approximation. Then, two one-dimensional diabatic PESs were constructed, one where the $\mathrm{O}_{2}$ molecule was kept neutral and one where a full electron was transferred [11]. This description could indeed account for the qualitative shape of the experimental sticking curve, as could the method by Binetti et al. [12], who followed a comparable approach but considered four different diabatic model PESs. Both treatments therefore point at the possible importance of nonadiabatic effects, but due to the arbitrary and severe approximations doubts remain about their conclusiveness.

Our works starts from recognizing that chemical interactions are ruled by various selection rules, and for the present situation spin-conservation [13] is expected to be relevant. In gas-phase chemistry it is well known that $\mathrm{O}_{2}$, when in its triplet ground state, is rather inert when the other reactant and the product are spin singlets. Interestingly, this role of the $\mathrm{O}_{2}$ spin has not attracted much attention in the $\mathrm{O}_{2} / \mathrm{Al}(111)$ interaction, although it was, e.g., studied for the adsorption of oxygen on $\mathrm{Si}(100)$ [14]. The appropriate theoretical modeling should then constrain the spin to the $\mathrm{O}_{2}$ Hilbert subspace, preventing charge transfer, as well as spin quenching before the systems interact. Such a spin-constrained DFT approach has neither been formulated nor evaluated for moleculesurface scattering so far. We will show that it not only gives a good description of the sticking coefficient (cf. Fig. 1, open circles), but may also explain the enigmatic abstraction mechanism.

Let us briefly describe the theoretical method enabling us to study the dynamics of an $\mathrm{O}_{2}$ molecule that remains in its spin-triplet configuration. Only very close to the surface are transitions to other configurations of the $\mathrm{O}_{2} / \mathrm{Al}(111)$ system able to set in. In order to calculate the spin-triplet PES we follow the work of Dederichs et al. [15], for which one must first define the Hilbert subspace of the $\mathrm{O}_{2}$ molecule. As the $\mathrm{DMol}^{3}$ code employs an atom-centered basis set, we use for this all basis functions that are also needed to provide a good description of the free $\mathrm{O}_{2}$ molecule. Then, for any position of the $\mathrm{O}_{2}$ molecule, we request that the total electron spin in this Hilbert subspace is one. In practice, this approach involves the self-consistent filling of the four partial densities of states of the spin-up and spin-down, $\mathrm{O}_{2}$ and $\mathrm{Al}(111)$ subsystems. This is formulated in terms of an auxiliary field in order to properly include the effect of the spin-constraint on the total energy [7].

Before discussing the results obtained with this approach, we recall two general problems of present-day Kohn-Sham DFT: First, even with gradient corrected XC functionals the description of the binding energy of the free $\mathrm{O}_{2}$ molecule is rather bad. Going from the $\mathrm{O}_{2}$ spin-triplet ground state to two free oxygen atoms, each of them also in the spin-triplet ground state, the errors of our calculated binding energies with respect to the experimental value (5.1 $\mathrm{eV} \mathrm{[16])} \mathrm{are} \mathrm{as} \mathrm{follows:} 2.3 \mathrm{eV}$ (local-density approximation), $1.0 \mathrm{eV}$ (PBE), $0.6 \mathrm{eV}$ (Becke-Lee-Yang-Parr), and $0.5 \mathrm{eV}$ (RPBE). Fortunately, for the part of the PES that is important for the sticking coefficient, we find that different functionals give results that differ by much less, indicating some favorable error cancellation. Below we will therefore restrict our discussion to the PBE and the RPBE, since they represent the extreme cases for the gradient corrected functionals, yielding the strongest and smallest overbinding in the $\mathrm{O}_{2}$ molecule, respectively. A second noteworthy problem arises because the expectation value of $S^{2}$ is not defined in Kohn-Sham DFT. For the present case this implies that the multiplet structure is not well described $[17,18]$. In free $\mathrm{O}_{2}$ the many-body ground state belongs to the triple degenerate ${ }^{3} \Sigma_{\mathrm{g}}^{-}$state which is followed by two 
singlets, namely, a doubly degenerate ${ }^{1} \Delta_{\mathrm{g}}$ level $(0.98 \mathrm{eV}$ above the ground state) and a nondegenerate ${ }^{1} \Sigma_{\mathrm{g}}^{+}$level (1.63 eV above the ground state). While the total energy of the spin-triplet ground state is described well, the ${ }^{1} \Delta_{\mathrm{g}}$ and ${ }^{1} \Sigma_{\mathrm{g}}^{+}$states are not described appropriately, since here DFT with jellium-based XC functionals describes a certain mixture of multiplets. A reasonable approximation to the true spin-singlet state is instead obtained by a spinunpolarized calculation [7], which for PBE (RPBE) is $1.1 \mathrm{eV}(1.2 \mathrm{eV})$ higher than the spin-triplet ground state.

Figure 2 shows two cuts through the calculated sixdimensional PESs for three situations: the adiabatic approximation (discussed in the introduction), the spin-triplet PES (using constrained DFT), and the spin-unpolarized calculation (which is the best we can do to describe the spin-singlet PES). Whereas the two elbow plots of the adiabatic PES (cf. Fig. 2, left panels) do not exhibit sizeable energy barriers toward dissociative adsorption, we find clear barriers on the triplet PES (cf. Fig. 2, middle panels). In fact, inspecting the whole six-dimensional triplet PES there is always an energy barrier (the lowest one is $0.05 \mathrm{eV}$ ). The right panels of Fig. 2 show the corresponding cuts through the singlet PES, which never exhibits any energy barriers. Clearly, an $\mathrm{O}_{2}$ molecule prepared in the singlet state would therefore react most efficiently with the $\mathrm{Al}(111)$ surface. Since the spin forbidden transition to the triplet ground state can proceed only by scattering with another molecule, the long lifetime of a singlet $\mathrm{O}_{2}$ should render molecular beam experiments possible to verify this proposition.

The sticking coefficient for these PESs is calculated as described above, i.e., using the "divide and conquer" approach [6-8]. The results for the adiabatic and the triplet PESs, using the RPBE functional, are given in Fig. 1. Obviously, the spin-triplet PES gives a sticking curve in good agreement with the experimental result. However, when the $\mathrm{O}_{2}$ and $\mathrm{Al}(111)$ wave functions overlap at close distances, spin transfer will occur with a certain probability. Because of the uncertainty in the description of the singlet PES, it is at present not very meaningful to perform a quantitative evaluation of these transition probabilities. A rough estimate of the importance of transitions bringing the system away from the triplet PES is instead provided by the width of the $2 \pi^{*}$ Kohn-Sham resonance, which is the level that carries the spin. At large distance the width is zero, and it gradually increases upon approach to the surface. For a one-dimensional cut through configuration space this is shown in Fig. 3. The peak width remains quite
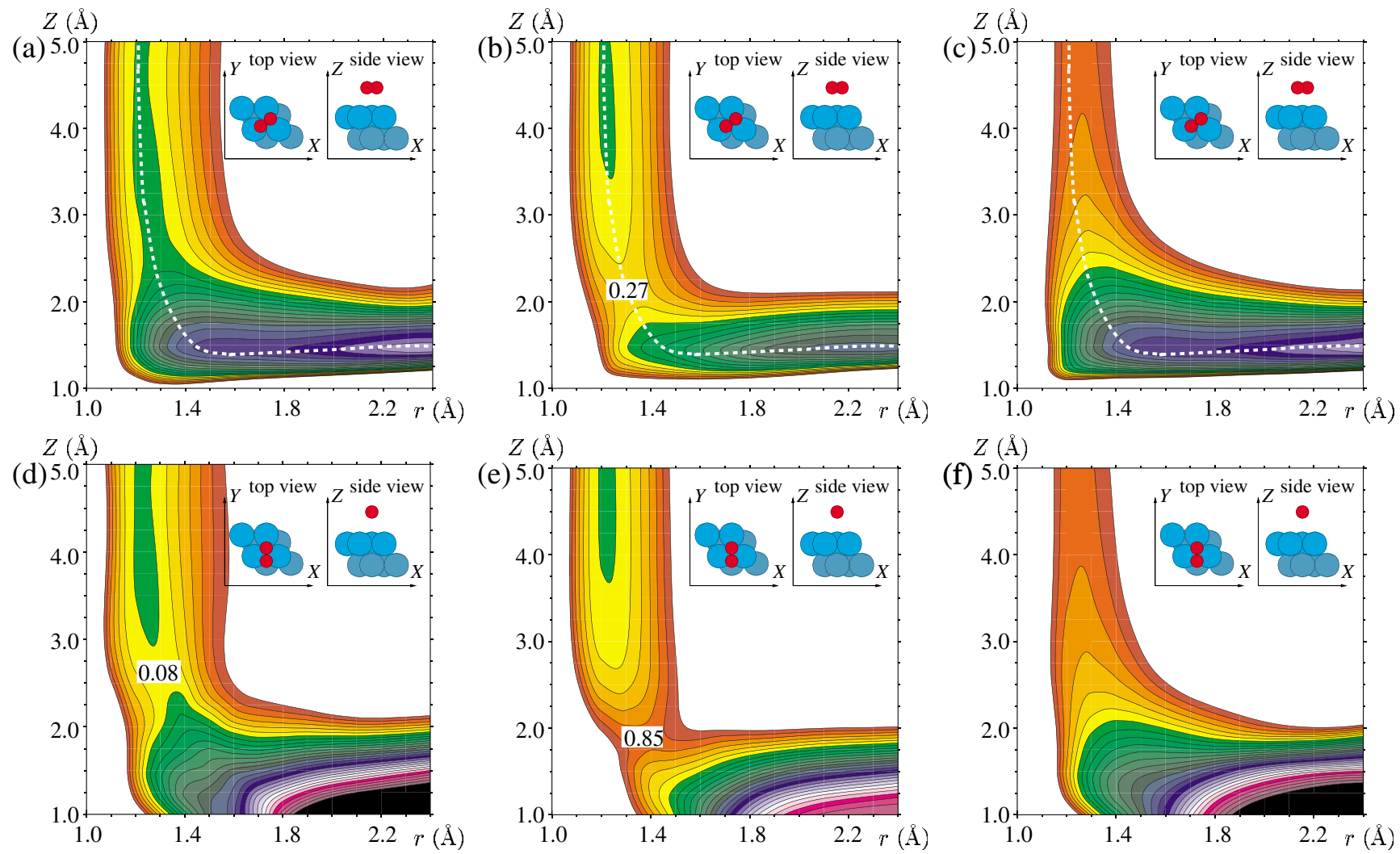

FIG. 2 (color online). Two-dimensional (elbow) cuts through the six-dimensional PESs calculated for three different situations, always using DFT RPBE (see text): adiabatic (a),(d), triplet (b),(e), and singlet PES (c),(f). The energies are shown as a function of the $\mathrm{O}_{2}$ bond length $r$ and of the distance $Z$ of the $\mathrm{O}_{2}$ center of mass from the surface. The angles and lateral positions are indicated in the insets. The energy zero corresponds to a free triplet $\mathrm{O}_{2}$ molecule. Contour lines are drawn at 0.2 eV intervals. Dissociation barriers (if present) are labeled (eV). 


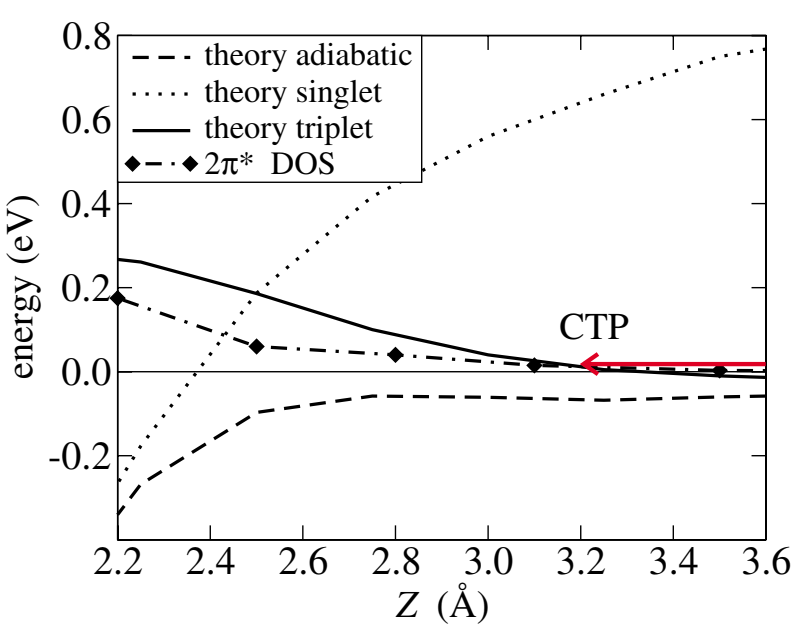

FIG. 3 (color online). Potential energy along the reaction path shown as a dashed line in Figs. 2(a)-2(c) (solid line is the triplet PES, dotted line is the singlet PES, and dashed line is the adiabatic PES). The arrow indicates the classical trajectory of a thermal $\mathrm{O}_{2}$ molecule constrained to the triplet PES, with CTP marking the classical turning point. At this point the coupling, represented by the width of the O $2 p$ Kohn-Sham level (dashdotted line) in the density of states (DOS), is only just emerging.

narrow, and even at the point where the triplet and singlet PESs cross it is only about $0.1 \mathrm{eV}$. In general, the lifetime of the $2 \pi^{*}$ electrons should be compared to the time the molecule spends between the classical turning point (CTP) and ca. $5 \AA$ away from the surface. For thermal molecules (cf. the arrow and the CTP point in Fig. 3) the comparison is lifetime $\approx 3$ ps versus time of presence $\approx 1 \mathrm{ps}$. We therefore conclude that for thermal $\mathrm{O}_{2}$ molecules (and even for all molecules with a kinetic energy below $\sim 0.2 \mathrm{eV}$ ) transitions away from the triplet PES will not play a big role. Our results then suggest that particularly these lowest energy molecules should be repelled by the barriers on the triplet PES, well before there is significant hybridization of wave functions, i.e., before relaxation towards the adiabatic ground state occurs. Only for higher kinetic energies will transitions gradually become important, leading to higher sticking coefficients than in the theory-triplet curve shown in Fig. 1. We also note that the PESs of the PBE and RPBE functionals are similar, but quantitative differences exist. These differences have noticeable influence on the calculated sticking curve only for kinetic translational energies below $0.2 \mathrm{eV}$. As the RPBE gives a better description for free $\mathrm{O}_{2}$ we place a higher credibility on its PES. Details will be discussed elsewhere [7].

Analyzing the approaching $\mathrm{O}_{2}$ molecule in greater detail reveals finally another interesting feature. For molecules that approach in an orientation perpendicular to the surface (or close to this) the spin is shifted to the atom that is further away from the surface. We believe this to be the onset of adsorption by the abstraction mechanism. In this way, one $\mathrm{O}$ atom can adsorb in a singlet state, while the spin is efficiently carried away with the other $\mathrm{O}$ atom that is either repelled back into the vacuum or to a distant place at the surface. Calculating the full dynamics of this process, i.e., going beyond the onset of dissociation important for the sticking coefficient, requires the explicit consideration of forces on the $\mathrm{Al}$ atoms, which we are implementing at present.

In summary, we have shown that spin selection rules can play an important role for $\mathrm{O}_{2}$ scattering at metals. They imply that $\mathrm{O}_{2}$ molecules should travel in a spin-triplet configuration up to distances close to the surface where hybridization with metal-surface states becomes significant. This is particularly important for systems with a low density of states at the Fermi level; for transition metals we expect that the high density of $d$ states at the Fermi level can more easily take up the spin. At $\mathrm{Al}(111)$ spin selection leads to a very low sticking probability for thermal $\mathrm{O}_{2}$ molecules in the triplet ground state, while $\mathrm{O}_{2}$ molecules prepared in the singlet configuration should adsorb with high probability. Similar effects as those discussed in this Letter should just as well play a role for other substrates with a low jelliumlike density of states at the Fermi level, and for other molecules with a high-spin ground state.

[1] L. Österlund, I. Zorić, and B. Kasemo, Phys. Rev. B 55, 15452 (1997).

[2] H. Brune et al., Phys. Rev. Lett. 68, 624 (1992).

[3] K. Honkala and K. Laasonen, Phys. Rev. Lett. 84, 705 (2000); Y. Yourdshahyan, B. Razaznejad, and B. I. Lundqvist, Phys. Rev. B 65, 75416 (2002).

[4] A. J. Komrowski et al., Phys. Rev. Lett. 87, 246103 (2001).

[5] $\mathrm{DMol}^{3}$ academic version [B. Delley, J. Chem. Phys. 92, 508 (1990)]. Employed basis set: 9 bohr real space cutoff for the basis functions, "all" basis set for O and "dnd" basis set for $\mathrm{Al},(4 \times 4 \times 1)$ Monkhorst-Pack $k$ mesh.

[6] S. Lorenz, A. Groß, and M. Scheffler, Chem. Phys. Lett. 395, 210 (2004); S. Lorenz, Ph.D. thesis, Technical University of Berlin, 2001.

[7] J. Behler et al. (to be published).

[8] A. Groß and M. Scheffler, Phys. Rev. B 57, 2493 (1998).

[9] J. P. Perdew, K. Burke, and M. Ernzerhof, Phys. Rev. Lett. 77, 3865 (1996).

[10] B. Hammer, L. B. Hansen, and J. K. Nørskov, Phys. Rev. B 59, 7413 (1999).

[11] A. Hellman et al., Surf. Sci. 532-535, 126 (2003).

[12] M. Binetti et al., Chem. Phys. Lett. 373, 366 (2003).

[13] E. Wigner, Nachr. Ges. Wiss. Goett., Math.-Phys. Kl., 375 (1927).

[14] K. Kato, T. Uda, and K. Terakura, Phys. Rev. Lett. 80, 2000 (1998).

[15] P. H. Dederichs et al., Phys. Rev. Lett. 53, 2512 (1984).

[16] G. Herzberg, Can. J. Phys. 30, 185 (1952).

[17] O. Gunnarsson and R. O. Jones, J. Chem. Phys. 72, 5357 (1980).

[18] U. von Barth, Phys. Rev. A 20, 1693 (1979). 